\title{
A 48-year-old man with chronic right shoulder pain and weakness after a fall: diagnosis and discussion
}

\author{
Matthew D. Epstein • Puneet Bhargava • \\ Jonathan R. Medverd
}

Published online: 3 February 2010

(C) ISS 2009

\section{Diagnosis}

Post-traumatic chronic axillary nerve injury (Fig. 1).

\section{Discussion}

Rotator cuff tears following shoulder dislocation are the most common cause of prolonged shoulder pain. However, 9 to $18 \%$ of patients who have anterior dislocation actually suffer from prolonged pain due to axillary nerve injury [1].

The axillary nerve $(\mathrm{C} 5, \mathrm{C} 6)$ is a branch of the posterior cord of the brachial plexus and exits the axilla to enter the quadrilateral space. This space is bounded by the teres minor muscle superiorly, the teres major muscle inferiorly, the long head of the triceps muscle medially, and the surgical neck of the humerus laterally. The anterior branch of the axillary nerve supplies the anterior deltoid muscle

The case presentation can be found at doi:10.1007/s00256-009-0846-Z.

M. D. Epstein

Department of Radiology, University of Washington,

1959 NE Pacific Street, Box 357115, Seattle, WA 98195-7115, USA

P. Bhargava $(\square) \cdot J$. R. Medverd

Department of Radiology, University of Washington,

1660 S Columbian Way, S-114/Radiology, Mail Stop 358280,

Seattle, WA 98108, USA

email: bhargp@u.washington.edu and overlying skin, while the posterior branch supplies the teres minor muscle, the posterior deltoid muscle, the skin overlying the distal deltoid muscle, and proximal triceps [2]. In an anterior shoulder dislocation, axillary nerve injury presumably occurs because of inferior displacement of the humeral head causing compression of the quadrilateral space [1].

Clinical symptoms of axillary nerve injury include vague shoulder pain, decreased strength on abduction, and abnormal sensation in the cutaneous distribution of the axillary nerve.

The definitive diagnostic test used to confirm the clinical diagnosis of axillary nerve injury is electromyography (EMG). However, EMG is painful and operatordependent. MR imaging, on the other hand, is a noninvasive technique that can help provide a diagnosis, especially when other diagnoses besides axillary nerve injury are being considered [3].

Anatomical distribution of the signal changes on MR distinguishes nerve injury from direct traumatic injury of muscles. With axillary nerve injury, some or all of the muscles supplied by the axillary nerve will be affected, depending on the location of the injury along the path of the axillary nerve [1]. MR signal from acute denervation within the first $24 \mathrm{~h}$ may manifest as increased short tau inversion recovery (STIR) signal and as increased apparent diffusion coefficient (ADC) [4, 5]. Absence of edema of overlying soft tissues allows distinction of acute denervation from acute traumatic muscle injury. Subacute muscle denervation causes uniform muscle edema in approximately $2-4$ weeks. Shifting of water from the intracellular to the extracellular space causes increased MR signal in the fluid-sensitive 
Fig. 1 a Proton density ( $P D$; repetition time $\mathrm{ms} /$ echo time $\mathrm{ms}$, $1,618 / 20$ ) sequence coronal oblique image; b T2-weighted spectral presaturation with inversion recovery (SPIR; repetition time $\mathrm{ms} / \mathrm{echo}$ time $\mathrm{ms}$, $3,142 / 60$ ) sequence coronal oblique image; c, d T2-weighted (repetition time ms/echo time ms, 1,542/62) sequence sagittal oblique images. Increased signal and fatty atrophy is seen in the deltoid (white arrows) and the teres minor (black arrows). Sup supraspinatus muscle, $I$ infraspinatus muscle, $S u b$ subscapularis muscle
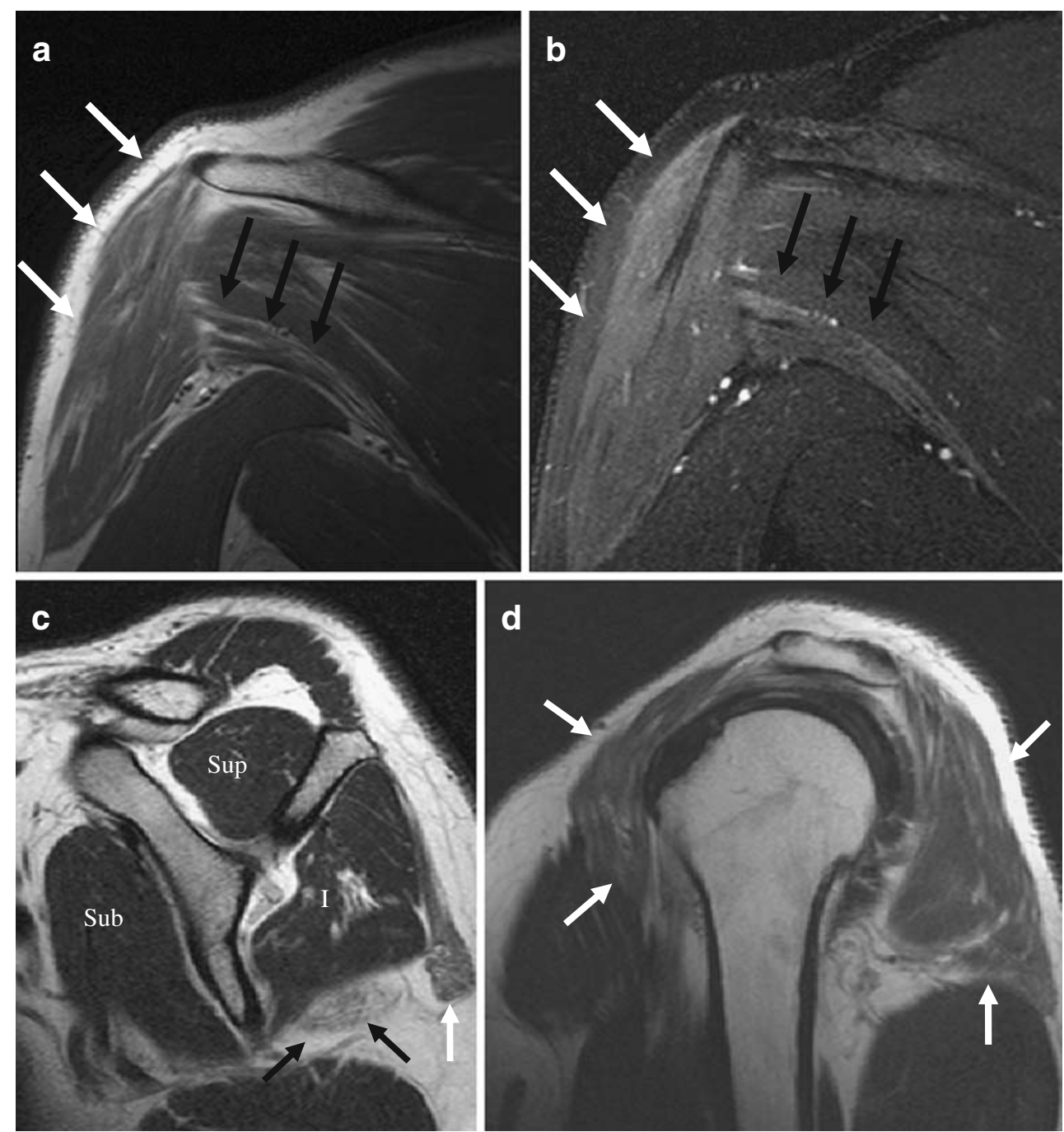

sequences. If normal innervation is restored, MR findings ultimately return to normal. However, if innervation is not restored, atrophy with fatty infiltration develops after a period of months, indicating irreversible muscle injury [6]. Our case illustrates this MR appearance of chronic axillary nerve injury.

Axillary nerve injury can also occur with fracture of the proximal humerus or a stretching injury of the brachial plexus. A nontraumatic cause of axillary nerve injury is quadrilateral space syndrome, caused by axillary nerve compression from fibrous bands in the quadrilateral space, which produces selective denervation of the teres minor muscle, with or without denervation of the deltoid muscle. Following surgical lysis of these bands, clinical improvement often ensues [7].

Identification of MR changes of chronic axillary nerve injury can prompt targeted management such as transcutaneous electrical nerve stimulation (TENS) and directed physiotherapy to strengthen deltoid and teres minor muscles [8].

\section{References}

1. Tuckman GA, Devlin TC. Axillary nerve injury after anterior glenohumeral dislocation: MR findings in three patients. AJR Am J Roentgenol. 1996;167:695-7.

2. Uetani M, Hayashi K, Matsunaga N, Imamura K, Ito N. Denervated skeletal muscle: MR imaging. Work in progress. Radiology. 1993;189:511-5

3. Fleckenstein JL, Watumull D, Conner KE, et al. Denervated human skeletal muscle: MR imaging evaluation. Radiology. 1993;187:213-8.

4. Bendszus M, Koltzenburg M, Wessig C, Solymosi L. Sequential MR imaging of denervated muscle: experimental study. AJNR Am J Neuroradiol. 2002;23:1427-31.

5. Holl N, Echaniz-Laguna A, Bierry G, et al. Diffusion-weighted MRI of denervated muscle: a clinical and experimental study. Skeletal Radiol. 2008;37:1111-7.

6. May DA, Disler DG, Jones EA, Balkissoon AA, Manaster BJ. Abnormal signal intensity in skeletal muscle at MR imaging: patterns, pearls, and pitfalls. Radiographics. 2000;20:S295-315.

7. Cormier PJ, Matalon TA, Wolin PM. Quadrilateral space syndrome: a rare cause of shoulder pain. Radiology. 1988;167:797-8.

8. Kazemi M. Acute traumatic anterior glenohumeral dislocation complicated by axillary nerve damage: a case report. J Can Chiropr Assoc. 1998;42:150-5. 